\title{
DESIGN OF A LOW-COST STEERABLE TEXTILE ARRAY OPERATING IN VARYING RELATIVE HUMIDITY CONDITIONS
}

\author{
Maria Lucia Scarpello, Dries Vande Ginste, Member, IEEE, Hendrik Rogier, Senior Member, IEEE
}

\begin{abstract}
A new wearable textile antenna array, operating in the $2.45 \mathrm{GHz}$ ISM band is described. Its purpose is to increase signal levels in off-body indoor communication links thanks to the array gain. In addition, multipath fading is reduced, thanks to its narrow beamwidth. As antenna substrate, we compare two textiles that have very different moisture regains, being cotton fabric and polyurethane foam and evaluate the array performance under varying relative humidity conditions in a controlled environment. Relative humidity has little influence on the mutual coupling, whereas for the reflection coefficients, the array constructed on foam material exhibits the most stable antenna characteristics.
\end{abstract}

Index Terms-Wearable antenna array, textile microstrip patch antenna, relative humidity, mutual coupling.

\section{INTRODUCTION}

Wearable textile antennas, combining textiles and electronics and integrated into garments, are conceived to be flexible, light weight, and conformable to their wearer [1]-[3]. They can be used for monitoring the activity and life signs of rescue workers active in a disaster area, as they allow to set up an off-body wireless communication link between the wearer and a base station [4]. The textile antenna array presented in this letter is intended to be part of a wireless body and personal area network, operating in the $2.45 \mathrm{GHz}$ Industrial, Scientific, and Medical (ISM) band (2.4-2.4835 GHz).Similar to a stand-alone textile antenna, the array must be comfortable to its wearer and withstand harsh environmental conditions, such as humidity. For example, as fire fighters are subjected to heat and heavy physical exertion, there is an increase of thermal sweating, which changes the relative humidity $(\mathrm{rH}) \mathrm{a}$ lot. In addition, the new proposed array exhibits some important advantages over a single textile patch, being significant array gain combined with a narrow main beam that is steerable in a small angular sector centered around broadside. Thereby, it will improve the reliability of wireless communication links thanks to increased signal levels and smaller fading fluctuations. A uniform linear array (ULA) topology, composed of four tiptruncated Equilateral Triangular Microstrip Patch Antennas (ETMPAs), is adopted. The choice of ETMPAs leads to low mutual coupling, while still providing sufficient gain in the broadside direction, i.e. away from the rescue worker and towards the base station. In contrast to non-uniform arrays, the presented ULA allows relatively easy steering and low mutual

Ghent University, Department of Information Technology (INTEC), Electromagnetics Group, Sint-Pietersnieuwstraat 41, B-9000 Gent, Belgium. e-mail marialucia.scarpello@ugent.be. coupling. Since the array beam is steered in a small angular sector centered around broadside, grating lobes are not an issue. Whereas in [5] the influence of $\mathrm{rH}$ on a single patch antenna has been described, in this letter, for the first time, the effect of $\mathrm{rH}$ on a textile antenna array is studied, keeping in mind the intended application. Thereto, the ULA is manufactured using two different non-conductive textile materials, i.e. cotton fabric and a polyurethane foam, called "Azzurri", manufactured by Brunet Lion [6]. These two substrates are chosen because they exhibit a very different Moisture Regain (MR), a material parameter defined in Section III that quantifies the capability of the substrates to trap moisture. The influence of $\mathrm{rH}$ on the antenna array is carefully assessed in a controlled environment (i) by measuring the reflection coefficients of each single patch and (ii) by measuring isolation between patches.To obtain stable antenna array performance in all operating conditions, a substrate with a low MR needs to be chosen, as otherwise, the matching of the individual array elements is affected by environmental conditions. First, in Section II, the design of the antenna array on the two different substrates is presented. Next, in Section III, the measurement procedure and results are shown, together with the array gain, simulated by applying a uniformly progressing phase factor. In Section IV, conclusions are summarized.

\section{Design of the Textile Antenna Array}

The antenna array is required to cover the $2.45 \mathrm{GHz}$ Industrial, Scientific, and Medical (ISM) band (2.4$2.4835 \mathrm{GHz}$ ). As antenna elements for the ULA, we opted for tip-truncated ETMPAs (Fig. 1).

A triangular topology is frequently used for the design of microstrip patch antennas, as they lead to radiation characteristics similar to a rectangular microstrip patch antennas while occupying a smaller area. By cutting off a triangle from one the tips, the size is even further reduced [7], [8]. Additionally, the mutual coupling between ETMPAs is lower than between rectangular patches.

The array is positioned vertically on the human torso, as shown in Fig. 2, in between two layers of garment, to avoid being directly in touch with the skin of the person. Given the size of a human torso, the available array aperture is about 50 $\mathrm{cm}$ in height. The vertical array allows to confine the energy within a narrow beam centered around the azimuth plane. The 
large antenna aperture together with its narrow beamwidth reduces multipath, resulting in less fading fluctuations. The ULA is composed by four ETMPAs that are equidistantly spaced by a distance of $0.75 \lambda, \lambda$ being the freespace wavelength at $2.45 \mathrm{GHz}$, approximately $122 \mathrm{~mm}$. Four patch elements, spaced $92 \mathrm{~mm}$ apart, is a convenient choice allowing to exploit the full vertical aperture. It is also based on a trade-off between gain characteristics and minimizing mutual coupling on the one hand and providing limited steering possibilities in a small angular sector around broadside without grating lobes on the other hand. In addition, the cost of the array and its steering electronics is quite low given the limited number of antenna elements. To ensure comfort to the wearer, the array fully consists of textile materials: both the patches and the ground plane of the array are made out of Flectron, a breathable and highly conductive electrotextile material, more specifically, a copper plated nylon fabric with a surface resistivity of less than $0.10 \Omega / s q$. Each ETMPA is excited by means of a $50 \Omega$ coaxial SMA feed, manually soldered. The two substrates, cotton fabric and Azzurri foam, have been characterized at $2.45 \mathrm{GHz}$. The dielectric characteristics of the substrates are listed in Tables [ and $\Pi$, together with the geometrical parameters of the patches. The latter are also indicated in Fig. 1. A top view of the complete array is shown in 2 . The array was hand cut, glued, and soldered.

Table I: Tip-truncated ETMPA on the Azzurri substrate: dielectric properties and patch dimensions.

\begin{tabular}{|ll|ll|}
\hline \multicolumn{2}{|c|}{ Patch (mm) } & \multicolumn{2}{c|}{ Substrate } \\
\hline$a$ & 60.1 & $\mathrm{~L}(\mathrm{~mm})$ & 480 \\
$b$ & 69 & $\mathrm{~W}(\mathrm{~mm})$ & 180 \\
$c$ & 8 & $\mathrm{~h}(\mathrm{~mm})$ & 3.55 \\
$d$ & 52.8 & & \\
Xfeed & 27.5 & $\epsilon_{r}$ & 1.19 \\
Yfeed & 10 & $\tan \delta$ & 0.003 \\
\hline
\end{tabular}

Table II: Tip-truncated ETMPA on the cotton substrate: dielectric properties and patch dimensions.

\begin{tabular}{|ll|ll|}
\hline \multicolumn{2}{|c|}{ Patch (mm) } & \multicolumn{2}{c|}{ Substrate } \\
\hline$a$ & 51.3 & $\mathrm{~L}(\mathrm{~mm})$ & 480 \\
$b$ & 53 & $\mathrm{~W}(\mathrm{~mm})$ & 180 \\
$c$ & 6.6 & $\mathrm{~h}(\mathrm{~mm})$ & 2.27 \\
$d$ & 45.8 & & \\
Xfeed & 20.8 & $\epsilon_{r}$ & 1.94 \\
Yfeed & 7.7 & $\tan \delta$ & 0.02 \\
\hline
\end{tabular}

\section{Measurements of Reflection Coefficients And} Mutual Coupling AND Simulation of the Gain

During the design of the antenna array, two criteria were imposed within the entire $2.45 \mathrm{GHz}$ ISM band:

$$
\begin{gathered}
\left|S_{i j}\right|<-10 \mathrm{~dB} \text { for } i=j, \\
\left|S_{i j}\right|<-15 \mathrm{~dB} \text { for } i \neq j, \\
i, j=1,2,3,4,
\end{gathered}
$$

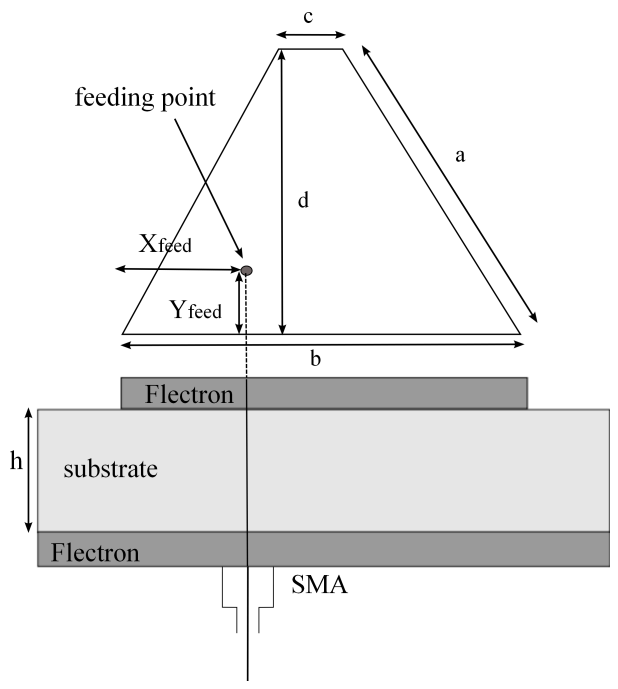

Figure 1: Side view of the textile antenna array and geometrical dimensions of a single ETMPA.
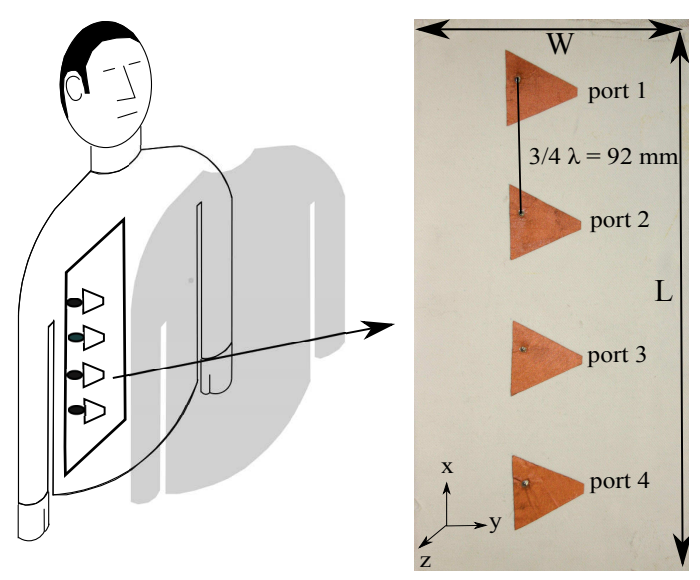

Figure 2: Top view of the textile antenna array and its position on the human body. On the right, there is a photograph of the array on the cotton substrate. Obviously, the other implementation on the foam substrate looks very similar.

where $i$ and $j$ indicate the port numbers of the antenna array. To assess the sensitivity of the array w.r.t. environmental conditions, more specifically, w.r.t. to changes in the relative humidity, two types of measurements are performed in a controlled environment and the results are verified against the conditions (1) and (2): first, the reflection coefficient of each individual array element is measured, while terminating the other three elements with a $50 \Omega$ load; second, the mutual coupling between each pair of antenna elements is measured, while terminating the other two elements with a $50 \Omega$ load.

Textile materials can absorb moisture, leading to changes of the electrical material parameters of the antennas, consequently influencing the antenna performance. The ISO standard 6741-4 [9] defines the Moisture Regain (MR) as the amount of moisture present in the material after 24 hours passive conditioning in standard conditions $\left(20^{\circ} \mathrm{C}\right.$ and $\left.65 \% \mathrm{rH}\right)$ compared to the amount present in dry state. Dry state is reached after drying for five hours in an oven at $105^{\circ} \mathrm{C}$. MR is calculated in \%, 
as follows:

$$
\mathrm{MR}=\frac{\text { Conditioned weight }- \text { Dry weight }}{\text { Dry weight }} \cdot 100 .
$$

The Azzurri and cotton substrate materials exhibit a small MR of $1.7 \%$ and a large MR of $6.9 \%$, respectively. To obtain reproducible and reliable measurements, the two implementations of the array were conditioned for 24 hours in a climate test cabinet (WK 350 from Weiss Technik) at a temperature of $23^{\circ} \mathrm{C}$ and the $\mathrm{rH}$ was varied each day. At the end of each day, they were taken out of the test cabinet, one by one, and immediately measured with an Agilent RF Network Analyzer placed next to the climate test cabinet to prevent any possible change in the conditions of the antenna.

Figs. $3 \mathrm{a}$ and $3 \mathrm{~b}$ show the measured reflection coefficients at port 1 of the arrays, when $\mathrm{rH}$ is set to be $10 \%, 30 \%$, $50 \%, 70 \%$, and $90 \%$. The array element on the Azzurri foam substrate exhibits a stable performance in terms of its reflection coefficient, always satisfying (1). The resonance frequency of the array element on the cotton substrate implementation, however, steadily decreases with increasing $\mathrm{rH}$. It is clear that for a $\mathrm{rH}$ of $70 \%$ and $90 \%$, condition (1) is violated and the ISM band is no longer covered. For the other antenna elements (ports 2-4) a similar behavior is observed. Figs. 3c $3 \mathrm{e}$ and $3 \mathrm{~g}$ show the mutual coupling between port 1 of the antenna array on Azzurri foam and the other three ports when the $\mathrm{rH}$ takes the following values: $10 \%, 30 \%, 50 \%, 70 \%$, and 90\%. In Figs. 3d, 3f and 3h results of the same experiment, using the array implemented on the cotton substrate, are shown. It is observed again that the implementation on the Azzurri foam is rather insensitive w.r.t. to environmental conditions, this in contrast to the implementation on the cotton substrate. However, thanks to the careful design, based on ETMPAs as single radiating elements combined with the ULA topology, the mutual coupling between the individual radiators remains low enough, and (2) is always satisfied for both implementations Similar characteristics are obtained for the other combinations of port pairs, i.e., 2 and 3, 2 and 4, 3 and 4. A change in $\mathrm{rH}$ will result in a varying dielectric constant of the substrate material together with a variation in substrate thickness, due to swelling of, e. g., the cotton fibers. These effects could be characterized by using techniques described in [10]. However, as also other effects could occur and to obtain a quick performance evaluation, in this letter we opted to immediately implement two designs and validating the end results by means of reliable measurements in a controlled environment. The gain pattern of the two textile arrays is simulated at $2.45 \mathrm{GHz}$ with the $2.5-\mathrm{D}$ EM field simulator Momentum of Agilent's Advanced Design System (ADS). This allows to check whether the distance between two ETMPAs causes undesirable grating lobes or not when performing beam steering with a limited angle sector of $20^{\circ}$ centered around broadside, as required by the application. The four ports are first excited with identical voltages. Next, a uniformly progressing phase factor is applied to steer the beam along $\theta_{m}=10^{\circ}$, where $\theta_{m}$ indicates the angle in the xz-plane where the gain is maximum. The simulated gain is reported in Fig. 4. The maximum gain for the array on Azzurri is $12.4 \mathrm{dBi}$ and the maximum gain for the array on cotton is $11.2 \mathrm{dBi}$.
The difference in magnitude between the main lobe and the first side lobe is more than $11 \mathrm{~dB}$ for both implementations, guaranteeing a good performance: the ULA, together with the ETMPA topology, offers high gain, easy beam steering, and low side lobes in the narrow angular sector, resulting in more reliable offbody communication links. The substrate is backed by a ground plane, so the two gain patterns are shown for half the azimuth plane, since the magnitude of the back lobes remains smaller than $-30 \mathrm{dBi}$.
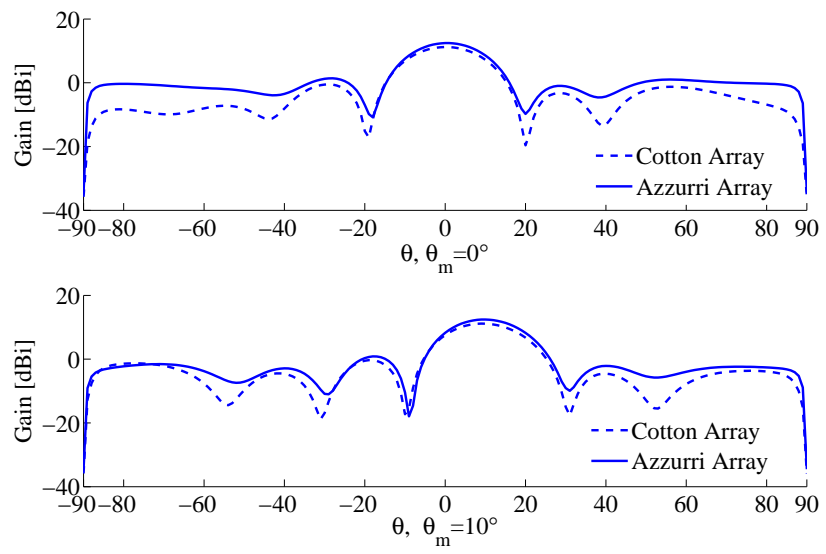

Figure 4: Gain pattern at $2.45 \mathrm{GHz}$ of the antenna array in the xz-plane, steering the beam along $\theta_{m}=0^{\circ}$ and $\theta_{m}=10^{\circ}$.

\section{Conclusion}

The design of a wearable antenna array implemented on two different non-conductive textile substrates, cotton fabric and Azzurri foam, and the study of its performance under varying the relative humidity conditions were presented. The array is intended to provide more reliable off-body communication links within the $2.45 \mathrm{GHz}$ ISM band and to be worn by rescue workers or fire fighters, active in a disaster area. Compared to a non-uniform array, the ULA guarantees low mutual coupling between the radiating elements, a narrow main beam, low side lobes, and relatively easy steering. In general, when choosing a textile material as a substrate for a textile antenna array to integrate into a garment, the MR value of the textile material must be taken into account. Here it was shown, under reproducible $\mathrm{rH}$ conditions realized in a climate test cabinet, that the array on Azzurri foam exhibits perfectly stable reflection coefficient characteristics of each individual antenna element. For the implementation on cotton, however, the resonance frequency decreases with increasing $\mathrm{rH}$, and the ISM band is no longer covered when the $\mathrm{rH}$ is above $70 \%$. Thanks to the ETMPA topology of the individual patches, the relatively small number of radiating elements, and the rather large spacing of $\frac{3}{4} \lambda$ between them, the mutual coupling is always low, even for the implementation on cotton, where the influence of $\mathrm{rH}$ is again noticeable. Hence, a low-cost array design with a large antenna aperture of about $50 \mathrm{~cm}$ and with limited steering capabilities centered around the broadside direction is obtained. When limiting the steering angle to $10^{\circ}$ around the broadside direction, no grating lobes appear. 


\section{REFERENCES}

[1] M. Klemm, I. Locher, G. Tröster, "A Novel Circularly Polarized Textile Antenna for Wearable Applications", Proc. of $7^{\text {th }}$ European Microwave Week, 2004, pp. 137-140.

[2] P. Salonen, H. Hurme, "A Novel Fabric WLAN Antenna for Wearable Applications", IEEE Antennas and Propagation Society International Symposium, 2003, pp. 700-703.

[3] C. Hertleer, A. Tronquo, H. Rogier and L. Van Langenhove, "The Use of Textile Materials to Design Wearable Microstrip Patch Antennas ", Textile Research Journal, Aug. 2008, vol. 78, n. 8, pp. 651-658.

[4] C. Hertleer, H. Rogier, L. Van Langenhove, "A Textile Antenna for Protective Clothing", 2007 IET Seminar on Antennas and Propagation for Body-Centric Wireless Communications, 2007, pp. 44-46.

[5] C. Hertleer, A. Van Laere, H. Rogier and L. Van Langenhove, "Influence of Relative Humidity on Textile Antenna Performance", Textile Research Journal, 2010, vol. 80, n. 2, pp. 177-183.

[6] http://lion-frankreich.lhd-gruppe.de/company.html.

[7] S. Sadat Karimabadi, Y. Mohsenzadeh, A. Reza Attari, and S. Mahdi Moghadasi, "Bandwidth Enhancement of Single-feed Circularly Polarized Equilateral Triangular Microstrip Antenna ", Progress in Electromagnetic research Symposium, Hangzhou China, Mar. 2008, pp. 147-150.

[8] C. Tang, J. Lu and K. Wong, "Circularly polarized Equilateral-Triangular Microstrip Antenna with Truncated Tip", Electronics Letters, Jun. 1998, vol. 34, n. 13, pp. 1277-1278.

[9] http://www.iso.org.

[10] F. Declercq, I. Couckuyt, H. Rogier, T. Dhaene, "Complex permittivity characterization of textile materials by means of surrogate modelling", IEEE International Symposium Antennas and Propagation and $\mathrm{CNC}$ USNC/URSI Radio Science Meeting, Jul. 2010. 


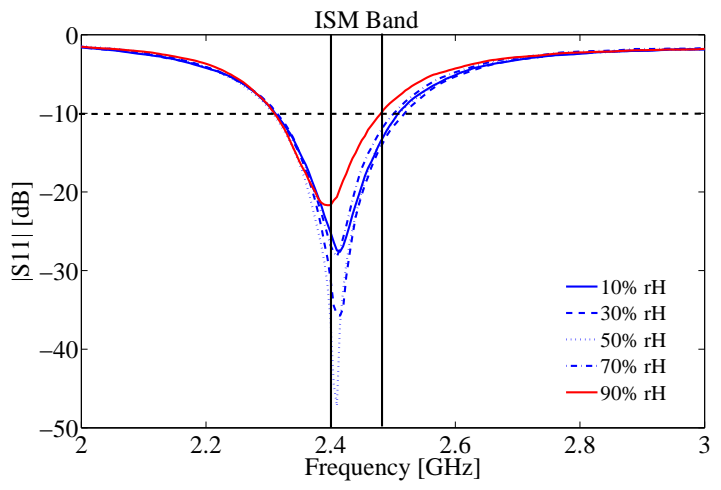

(a) Azzurri: reflection coefficient at port 1.

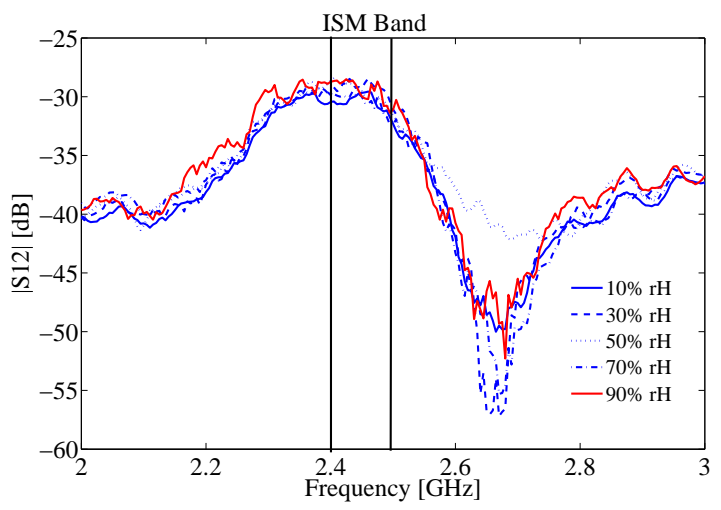

(c) Azzurri: mutual coupling between port 1 and 2.

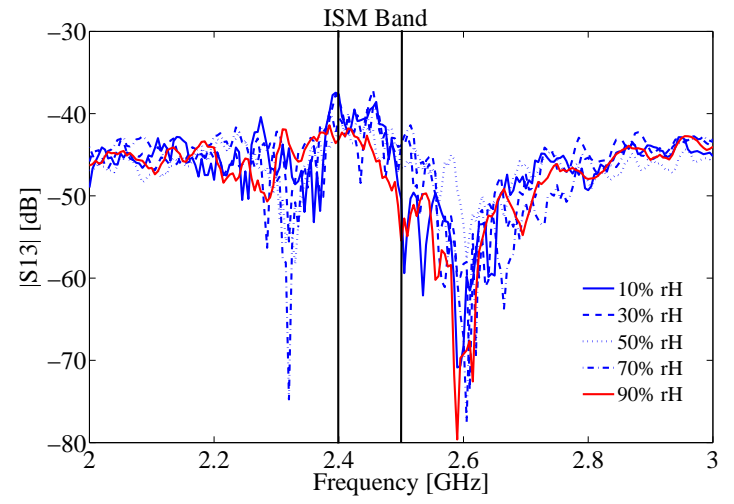

(e) Azzurri: mutual coupling between port 1 and 3 .

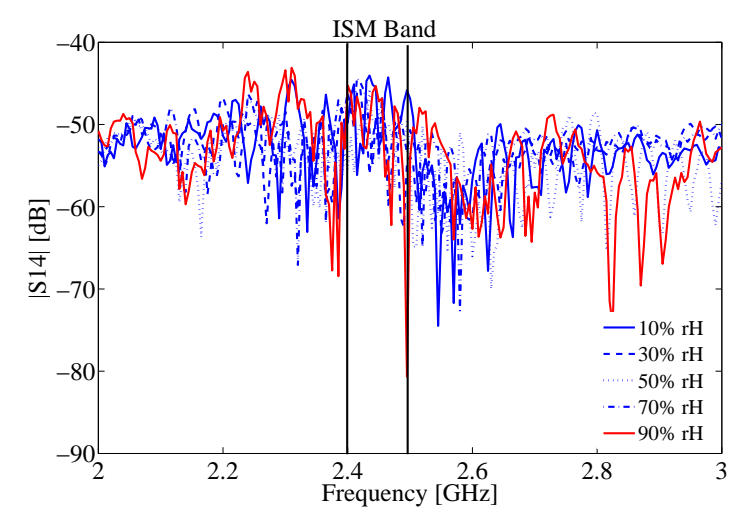

(g) Azzurri: mutual coupling between port 1 and 4 .

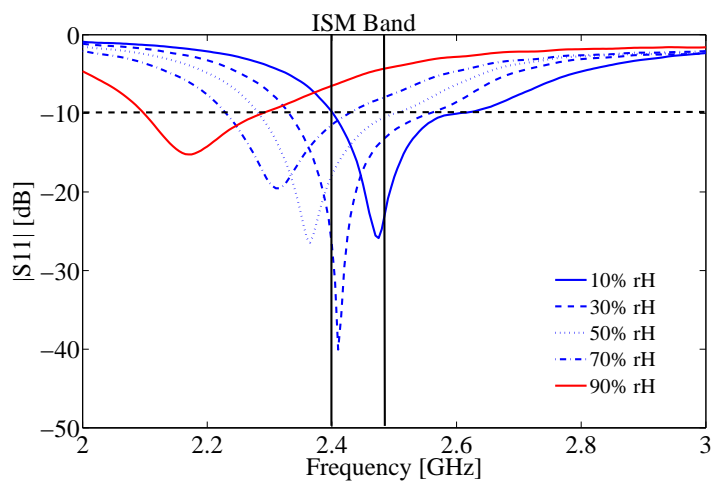

(b) Cotton: reflection coefficient at port 1 .

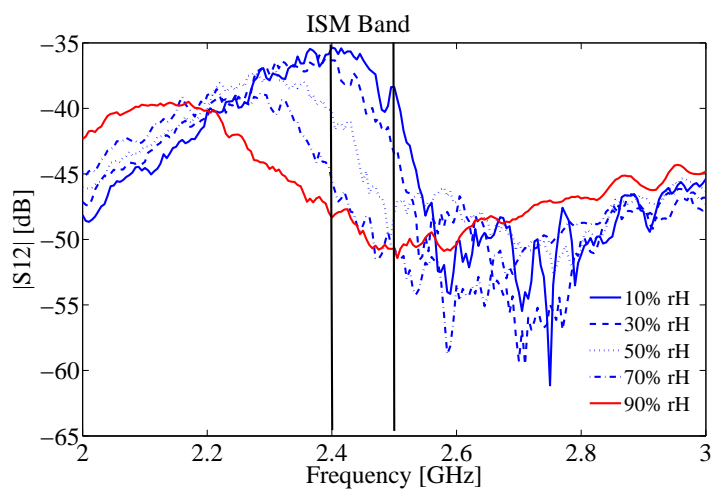

(d) Cotton: mutual coupling between port 1 and 2.

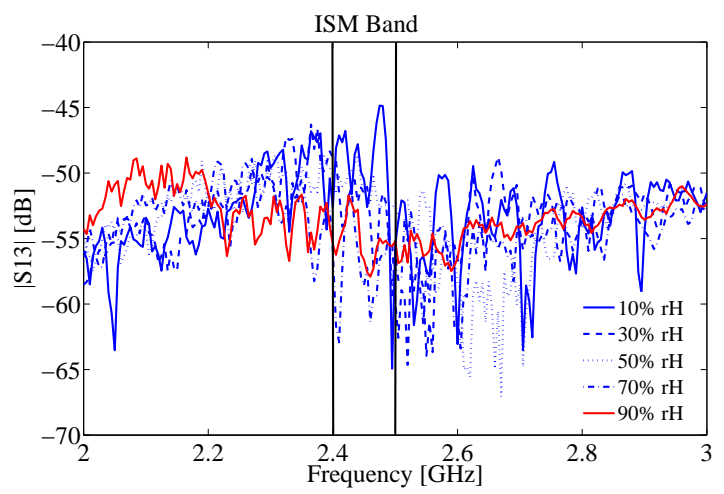

(f) Cotton: mutual coupling between port 1 and 3 .

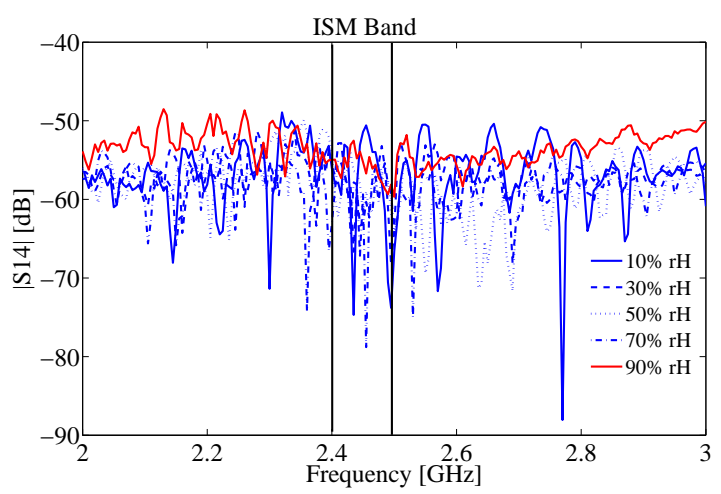

(h) Cotton: mutual coupling between port 1 and 4 .

Figure 3: S-parameters measurements after acclimatization of the array, for both implementations: reflection coefficients at port 1 of the array and mutual coupling between port 1 of the array and the other three ports. 